Tendências em Matemática Aplicada e Computacional, 3, No. 1 (2002), 71-80.

(C) Uma Publicação da Sociedade Brasileira de Matemática Aplicada e Computacional.

\title{
Metodologia para Representação de Modelos NARX Polinomiais na Forma de Hammerstein e Wiener
}

M.C.S. COELHO ${ }^{1}$, L.A. AGUIRRE ${ }^{2}$, Programa de Pós-Graduação em Engenharia Elétrica - UFMG, Av. Presidente Antônio Carlos, 6627, 31270-010 Belo Horizonte, MG, Brasil

M.V. CORRÊA ${ }^{3}$, Centro Universitário do Leste de Minas Gerais - UnilesteMG, Av. Presidente Tancredo Neves, 3500, Bairro Universitário, 35170-056 Coronel Fabriciano, MG, Brasil.

Resumo. Este trabalho apresenta uma metodologia para a obtenção de modelos de Hammerstein e Wiener a partir de um modelo NARX polinomial previamente identificado. Uma análise sobre a expansão polinomial dos modelos de blocos interconectados é realizada objetivando determinar algumas de suas propriedades. A aplicação desta metodologia a um Conversor CC-CC do tipo Buck é apresentada.

\section{Introdução}

Nas últimas três décadas consolidaram-se a teoria e a prática de identificação de sistemas. No início, os modelos matemáticos usados para representar os sistemas eram lineares e considerados suficientes apesar de não conseguirem reproduzir o comportamento dinâmico não-linear dos sistemas sob investigação. A maior parte da teoria e dos procedimentos adotados para projetar controladores para tais sistemas eram baseados nestes modelos lineares.

Com o avanço tecnológico e industrial, o interesse pela modelagem não-linear e o desenvolvimento de ferramentas matemáticas para entender melhor o comportamento dos fenômenos não-lineares cresceram significativamente, uma vez que as técnicas existentes para modelos lineares não conseguem reproduzir toda a gama de comportamentos dinâmicos dos sistemas reais.

Dentre as representações não-lineares podem-se destacar os modelos de blocos interconectados que representam o sistema através de blocos, sendo que em um dos blocos a dinâmica do sistema é representada por um modelo dinâmico linear e a não-linearidade por uma função estática não-linear. A grande vantagem de tal representação é a possibilidade de utilizar técnicas de identificação linear já então consolidadas. Os modelos de blocos interconectados foram muito utilizados até

\footnotetext{
${ }^{1}$ Agradecimentos à CAPES.

${ }^{2}$ Agradecimentos ao CNPq e à FAPEMIG

${ }^{3}$ Agradecimentos à UNILESTE.
} 
meados da década de oitenta. Naquela época, Billings e Leontaritis em [11] apresentaram os modelos NARMAX (Nonlinear Autoregressive Moving Average model with eXogenous inputs) polinomiais. Estes, por sua vez, são capazes de representar uma gama de sistemas não-lineares. Várias técnicas de seleção de estrutura e estimação de parâmetros foram desenvolvidas para esta representação. Apesar de ter sido usado na modelagem de diversos sistemas reais [1, 7], o desenvolvimento de controladores baseados nesta representação não experimentaram o mesmo avanço.

Em meados da década de noventa, o interesse por modelos de blocos interconectados ressurgiu. Um fator principal é a facilidade com que estes modelos podem ser tratados ao lidar com técnicas de controle [9]. Estes modelos podem ser aplicados também em processos químicos e em sistemas biológicos [10, 8].

Desta forma tem-se uma situação em que técnicas de identificação de modelos NARX polinomiais encontram-se bastante desenvolvidas [7], porém a utilização destas representações para projeto de controladores é relativamente limitada. Por outro lado, várias técnicas de controle baseadas em modelos de blocos interconectados encontram-se disponíveis [9], mas as técnicas para obtenção de tais modelos não apresentaram o mesmo avanço. Inúmeros artigos que tratam do problema de identificação de modelos de Hammerstein e de Wiener estão disponíveis na literatura, dentre os quais pode-se citar $[3,8,12]$.

Este trabalho propõe uma metodologia para obtenção de modelos de Hammerstein e Wiener a partir de um modelo NARX polinomial já identificado e de dados de entrada e saída de um sistema dinâmico não-linear.

\section{Modelos NARMAX}

Os modelos baseados em equações de diferença constituem uma das mais importantes classes de modelos matemáticos de sistemas lineares ou não-lineares. $\mathrm{O}$ modelo NARMAX (Non-linear AutoRegressive Moving Average with eXogenous inputs) $[5,11]$, com estrutura monovariável e período de amostragem normalizado, é dado por:

$$
\begin{aligned}
y(k)= & F^{l}\left[y(k-1), \ldots, y\left(k-n_{y}, u(k-d), \ldots, u\left(k-d-n_{u}\right), e(k-1), \ldots,\right.\right. \\
& \left.\left.e\left(k-n_{e}\right)\right)\right]+e(k),
\end{aligned}
$$

sendo $y(k), u(k), e(k)$, a saída, a entrada e o ruído aditivo do sistema, respectiva-

mente, e $n_{y}, n_{y}, n_{e}$, os seus respectivos atrasos máximos. $d$ é o tempo morto do sistema.

A forma da função $F^{l}$ normalmente não é conhecida a priori. Assim, a dinâmica do sistema deve ser reconstruída utilizando-se uma aproximação para representar $F^{l}$. Possíveis aproximações para esta função são os modelos polinomiais e racionais. Neste trabalho, a aproximação utilizada é a polinomial.

Um subconjunto do modelo NARMAX que contém apenas a sua parte determinística é denominado modelo NARX (Non-linear AutoRegressive with eXogenous inputs ) e é representado por:

$$
y(k)=F^{\ell}\left[y(k-1), \ldots, y\left(k-n_{y}, u(k-d), \ldots, u\left(k-d-n_{u}\right)\right]+e(k) .\right.
$$


Uma característica deste tipo de representação não-linear é a sua capacidade de recuperar a característica estática de um sistema a partir de dados de entrada e saída, resultando em uma relação analítica. Estudos de como funções estáticas são mapeadas em modelos NARX polinomiais podem ser vistos em [2].

Em [6] é mostrado que os modelos de Hammerstein e de Wiener são casos particulares dos modelos NARX polinomiais.

O modelo dinâmico linear ARX, de ordem $n_{y}$, é um subconjunto do modelo NARX. Tomando-se $\ell=1$ na equação (2.2), tem-se

$$
y(k)=\sum_{i=1}^{n_{y}} \sigma_{i} y(k-i)+\sum_{j=1}^{n_{u}} \theta_{j} u(k-j) .
$$

\section{Modelos de Blocos Interconectados}

Modelos de blocos interconectados ${ }^{4}$ representam sistemas dinâmicos não-lineares através da conexão entre dois blocos, sendo que um destes representa a dinâmica linear e o segundo a não-linearidade estática. A ordem em que estes blocos estão conectados é que diferencia um modelo do outro, conforme ilustrado pela Figura 1. Esta diferença na conexão dos blocos define modelos com comportamento dinâmico diferente, como o modelo de Hammerstein e o de Wiener.

(a)

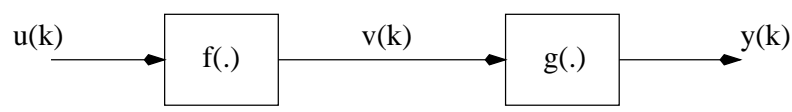

(b)

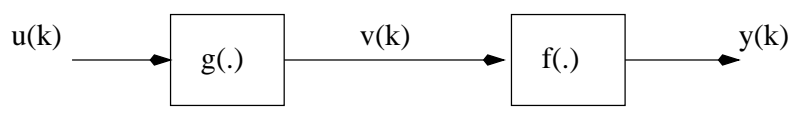

Figura 1: O modelo Hammerstein está ilustrado na figura (a) e o modelo Wiener na figura (b). $f($.$) é a função estática não-linear e g($.$) uma função dinâmica linear.$

No presente trabalho será considerado que o modelo que representa o bloco dinâmico linear é um modelo ARX dado pela equação (2.3), e a não-linearidade estática é representada por uma função não-linear polinomial ou racional.

\footnotetext{
${ }^{4}$ Um resumo sobre as principais metodologias já publicadas para identificação de modelos de Wiener e Hammerstein pode ser encontrado em $[3,8]$.
} 


\section{Propriedades dos Modelos de Hammerstein e Modelos de Wiener}

Esta seção deriva propriedades das representações de Hammerstein e de Wiener e suas conexões com modelos NARX polinomiais [6].

\subsection{Propriedades dos modelos de Hammerstein}

Observando a Figura 1(a) verifica-se que o sinal intermediário $v(k)$ é obtido pelo mapeamento do sinal de entrada $u(k)$ através da função $f$. Logo, tem-se que

$$
\begin{array}{r}
v(k)=f(u(k)), \\
v(k-q)=f(u(k-q)) .
\end{array}
$$

O modelo ARX é dado para o par de entrada e saída do bloco dinâmico linear, tal como mostra a equação (4.2),

$$
y(k)=\sum_{p=1}^{n_{y}} \theta_{p} y(k-p)+\sum_{q=1}^{n_{v}} \sigma_{q} v(k-q) .
$$

Substituindo a equação (4.1) em (4.2), o modelo de Hammerstein pode ser escrito na forma de um modelo NARX polinomial tal como

$$
y(k)=\sum_{p=1}^{n_{y}} \theta_{p} y(k-p)+\sum_{q=1}^{n_{u}} \sigma_{q} f(u(k-q)) .
$$

A equação (4.3) revela que o o modelo de Hammerstein é um caso particular da representação NARX polinomial em que:

- a não-linearidade está expressa em função de $u$;

- não há presença de termos do tipo $u(k-i) u(k-j)$, para $i \neq j$.

Chamando $\bar{u}$ e $\bar{y}$ de entrada e saída em estado estacionário, pode-se assumir que $\bar{u}=u(k)=u(k-1)=\ldots=u(k-q)$ e $\bar{y}=y(k)=y(k-1)=\ldots=y(k-p)[1,7]$. Utilizando este conceito na equação (4.3), a saída $\bar{y}$ pode ser expressa em função de $\bar{u}$, tal como

$$
\bar{y}=\frac{\left(\sum_{q=1}^{n_{u}} \sigma_{q}\right) f(\bar{u})}{1-\left(\sum_{p=1}^{n_{y}} \theta_{p}\right)} .
$$

Pela equação (4.4) verifica-se que modelos de Hammerstein possuem apenas uma saída em estado estacionário para cada valor da entrada estacionária $\bar{u}$ correspondente. Porém, se $f($.$) não for inversível, pode-se ter dois valores de entrada \bar{u}$ que levam a saída para o mesmo estado estacionário, $\bar{y}$. Portanto, a representação de Hammerstein, em estado estacionário, admite multiplicidade de entradas e apenas uma saída [12]. 


\subsection{Propriedades dos modelos de Wiener}

Observando a Figura 1(b) verifica-se que o sinal de saída do modelo Wiener é obtido pelo mapeamento do sinal intermediário $v(k)$ através da função $f$, da seguinte maneira,

$$
y(k)=f(v(k)) .
$$

Tendo em vista que o sinal intermediário $v(k)$ não pode ser medido, podese estimá-lo através da inversa da função $f$. Portanto, a função $f$ terá que ser inversível $^{5}$ para que um modelo Wiener possa ser estimado. Pela equação (4.5), obtém-se a seguinte relação,

$$
v(k)=\hat{f}^{-1}(y(k)) .
$$

O modelo ARX é determinado para o par de entrada e saída do bloco dinâmico linear, $u(k)$ e $v(k)$, respectivamente,

$$
v(k)=\sum_{p=1}^{n_{v}} \theta_{p} v(k-p)+\sum_{q=1}^{n_{u}} \sigma_{q} u(k-q) .
$$

Substituindo a equação (4.5) em (4.7), tem-se

$$
y(k)=f\left(\sum_{p=1}^{n_{v}} \theta_{p} v(k-p)+\sum_{q=1}^{n_{u}} \sigma_{q} u(k-q)\right) .
$$

A equação (4.6) pode ser reescrita como

$$
v(k-p)=\hat{f}^{-1}(y(k-p)) .
$$

Substituindo a equação anterior em (4.8), o modelo NARX polinomial equivalente ao modelo de Wiener é obtido, em relação aos sinais de entrada e saída do sistema, $u(k)$ e $y(k)$, respectivamente,

$$
y(k)=f\left(\sum_{p=1}^{n_{y}} \theta_{p} \hat{f}^{-1}(y(k-p))+\sum_{q=1}^{n_{u}} \sigma_{q} u(k-q)\right) .
$$

Chamando $\bar{u}$ e $\bar{y}$ de entrada e saída em estado estacionário, pode-se assumir que $\bar{u}=u(k)=u(k-1)=\ldots=u(k-q)$ e $\bar{y}=y(k)=y(k-1)=\ldots=y(k-p)[1,7]$. Utilizando este conceito na equação (4.9), a saída $\bar{y}$ pode ser expressa como função de $\bar{u}$ por

$$
\bar{y}=f\left(\frac{\left(\sum_{q=1}^{n_{u}} \sigma_{q}\right) \bar{u}}{1-\left(\sum_{p=1}^{n_{y}} \theta_{p}\right)}\right) .
$$

\footnotetext{
${ }^{5}$ Para que uma função $f: A \rightarrow B$ admita a inversa $f^{-1}$, é necessário que esta função $f$ seja bijetora. Sendo assim, tem-se $f^{-1}: B \rightarrow A$.
} 
Pela equação (4.10) verifica-se que modelos de Wiener admitem apenas uma saída estacionária, $\bar{y}$, para cada entrada correspondente, $\bar{u}$. Porém, dependendo de $f^{\ell}($.$) , este modelo pode assumir multiplicidade da entrada em estado estacionário { }^{6}$. A relação dada em (4.10) caracteriza a curva estática do modelo Wiener para o ponto de operação $(\bar{u}, \bar{y})$.

Além disso, a não-linearidade atua nos regressores da entrada e da saída Portanto, é possível a presença de termos cruzados do tipo $y(k-j) u(k-i)$. Sendo assim, modelos NARX que contenham termos cruzados em $u$ e $y$ e não-linearidades atuando em $y$, podem ser equivalentemente representados por um modelo Wiener.

\section{Metodologia}

A grande dificuldade para se obter as representações de Hammerstein e de Wiener está na necessidade de estimar as funções $f($.$) e g($.$) a partir de dados de entrada e$ saída, não tendo disponível o sinal intermediário $v(k)$.

Esta seção apresenta uma metodologia para se obter as representações de Hammerstein e de Wiener partir de dados de entrada e saída de um sistema dinâmico monovariável e de um modelo NARX polinomial previamente identificado. Esta justifica-se, conforme dito anteriormente, pelo grande número de modelos NARX polinomiais já identificados e pela relativa facilidade de extrair as características analíticas dos mesmos. No entanto, o que será utilizado do modelo NARX polinomial é a função que descreve a característica estática. A metodologia consiste dos seguintes passos:

1. Determinar a função estática não-linear $f$ a partir do modelo NARX polinomial e estimar o sinal intermediário $\hat{v}(k)$ utilizando os dados de entrada e saída do bloco estático.

- Se modelo Hammerstein, o sinal de entrada do bloco estático é o próprio sinal de entrada do sistema, $u(k)$.

- Se modelos de Wiener, a função $f$ tem que ser inversível. À $\hat{f}^{-1}$ é ajustado um polinômio de grau $n$. O sinal de entrada do polinômio é o sinal de saída do sistema, $y(k)$.

2. Identificar o modelo ARX a partir dos dados de entrada e saída do bloco dinâmico linear.

3. Validar os modelos.

\footnotetext{
${ }^{6} \mathrm{Se} f^{\ell}($.$) não admitir inversa os modelos de Wiener podem ter múltiplas entradas em estado$ estacionário [12]. Porém, neste trabalho, para que o modelo Wiener possa ser obtido, $f^{\ell}($.$) tem$ que ser inversível. Logo, os modelos de Wiener aqui considerados não possuem multiplicidade da entrada em estado estacionário.
} 


\section{Aplicações a um Conversor CC-CC Buck}

Nesta seção será apresentada a identificação de um conversor CC-CC do tipo Buck aplicando a metodologia proposta, objetivando encontrar um modelo de Hammerstein e um modelo de Wiener a partir dos dados de entrada e saída do sistema e da função não-linear extraída de um modelo NARX polinomial já identificado. Deseja-se que tais modelos sejam capazes de aproximar tanto a resposta dinâmica do sistema quanto a resposta estática fora da região dos dados de identificação. Os dados usados para identificação estão na Figura 2.
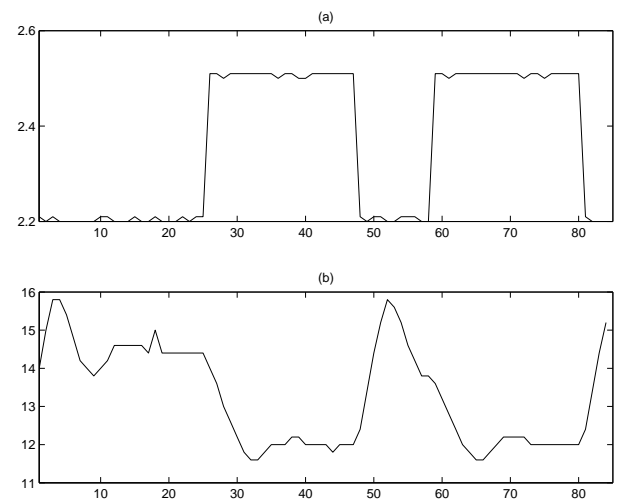

Figura 2: Em (a) estão os dados de entrada e em (b) os dados de saída usados na identificação. O eixo $x$ contém o número de amostras e o eixo $y$ a amplitude dos sinais em volts.

Teoricamente, a relação estática do conversor é dada por

$$
\text { vo }=\frac{4 v_{d}}{3}-\frac{v_{d}}{3} \bar{u}
$$

sendo $v_{d}$ a fonte CC de alimentação mantida constante em 24 volts.

O modelo NARX polinomial previamente identificado é dado por [7]

$$
\begin{aligned}
y(k)= & 0,7315 y(k-1)-0,0047 y(k-2)+13,7292-0,8280 u(k-1)^{3} \\
& -0,2495 y(k-3)+3,6774 u(k-3) u(k-1)^{2}+2,0210 u(k-3)^{3} \\
& -1,7617 u(k-1) u(k-3)-4,6409 u(k-1) u(k-3)^{2} .
\end{aligned}
$$

A característica estática do modelo NARX polinomial é dada pela relação (6.3)

$$
\bar{y}=0,4391 \bar{u}^{3}-3,3704 \bar{u}^{2}+26,2659 .
$$

Modelo Hammerstein: A função que descreve a característica es-tá-ti-ca do mo-de-lo NARX polinomial dada em (6.3) é reescrita para o par de entrada e saída do bloco estático não-linear de um modelo de Hammerstein tal como

$$
\bar{v}=0,4391 \bar{u}^{3}-3,3704 \bar{u}^{2}+26,2659 .
$$


O modelo ARX para o par de entrada e saída do bloco dinâmico linear do modelo Hammerstein, está descrito na equação (6.4),

$$
\begin{aligned}
y(k)= & 1,1016 y(k-1)+0,1323 y(k-2)-0,8710 y(k-3)+0,3520 y(k-4) \\
& +0,0145 y(k-5)-0,0090 y(k-6)-0,0241 y(k-7)+0,3449 v(k-1) \\
& -0,0383 v(k-2)-0,0984 v(k-3)+0,1416 v(k-4)-0,0455 v(k-5) .
\end{aligned}
$$

Modelo Wiener: A função estática não-linear dada em (6.3), é reescrita para o par de entrada e saída do bloco estático não-linear do modelo de Wiener, tal como:

$$
\bar{y}=0,4391 \bar{v}^{3}-3,3704 \bar{v}^{2}+26,2659,
$$

o polinômio de grau 3, ajustado à inversa da função descrita em (6.5), é dado por

$$
\bar{v}=-0,0005 \bar{y}^{3}-0,1048 \bar{y}^{2}+3,8296 .
$$

O modelo ARX obtido para o par de entrada e saída do bloco dinâmico linear do modelo de Wiener é descrito por

$$
\begin{aligned}
v(k)= & 0,8925 v(k-1)+0,1384 v(k-2)-0,2301 v(k-3) \\
& -0,1931 v(k-4)-0,0744 v(k-5)+0,1463 v(k-6) \\
& +0,3274 u(k-1)+0,0270 u(k-2)-0,0347 u(k-3) .
\end{aligned}
$$

Comparação entre os modelos: A característica estática obtida pela modelo NARX (equação (6.3)), está ilustrada na Figura 3.

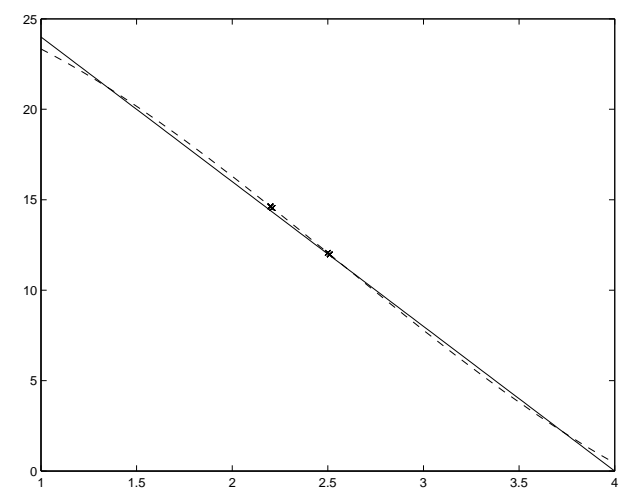

Figura 3: Característica estática. (-) Sistema e (- -) modelo NARX. O eixo $x$ é a entrada em regime permanente $\bar{u}$ e o eixo $y$ é a saída em regime permanente $\bar{y}$. As cruzes indicam a faixa de valores varrida pela entrada durante o teste dinâmico.

Salienta-se que a característica estática dos modelos de Hammerstein e de Wiener é a mesma do modelo NARX polinomial, para uma mesma entrada em estado estacionário, que varia entre $1 \leq \bar{u} \leq 4$. A validação dinâmica dos modelos NARX polinomial, Hammerstein e Wiener está ilustrada na Figura 4. 


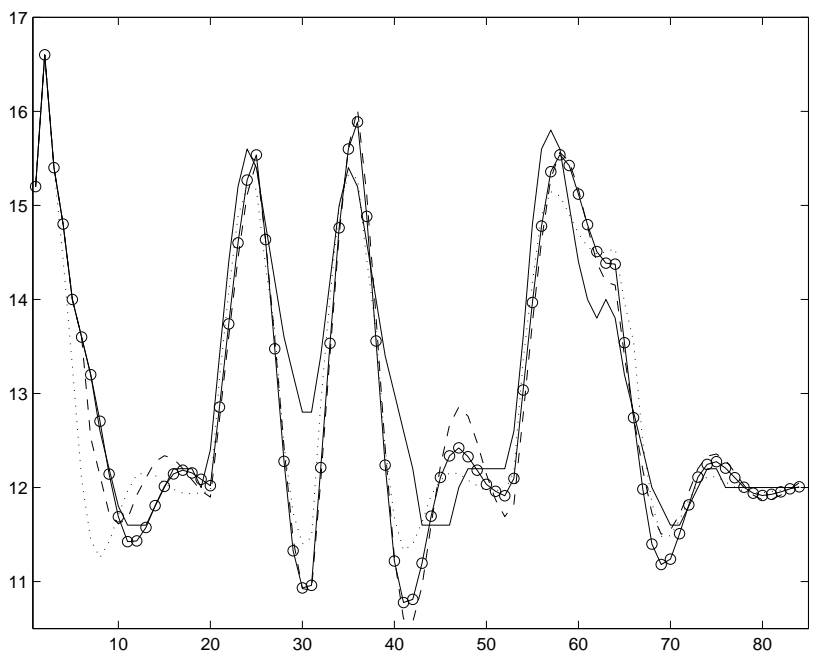

Figura 4: Validação dinâmica - Simulação livre. (-) Saída do sistema, (:) saída do modelo NARX, (- -) saída do modelo Wiener e (o-)e saída do modelo Hammerstein. O eixo $x$ contém o número de amostras e o eixo $y$ a amplitude do sinal em volts. O desempenho de cada representação é avaliado usando o índice RMSE, que para o modelo NARX, Hammerstein e Wiener é, respectivamente, 0,4294; 0,4312; 0,4753.

\section{Conclusão}

A metodologia apresentada é eficiente para obter modelos de Hammerstein e de Wiener a partir de dados de entrada e saída de um sistema e usando a função estática não-linear extraída de um modelo NARX polinomial previamente identificado. Verificou-se que um modelo de Hammerstein pode ser equivalente a um modelo NARX polinomial se este último não contiver termos cruzados entre $u$ e $y$, pois, a presença de tais termos acarreta em constante de tempo variável [7]. Além disso, tais modelos admitem apenas uma saída em estado estacionário.

Os modelos de Wiener admitem uma saída em estado estacionário e apresentam constante de tempo variável com o ponto de operação [7]. Portanto, estes modelos podem ser equivalentes a modelos NARX polinomiais que contenham termos cruzados em $u$ e $y$. Ressalta-se que nem todos os modelos NARX polinomiais podem ser equivalentemente representados por modelos de Hammerstein ou de Wiener.

Procedimentos para a identificação dos modelos de Hammerstein e de Wiener a partir de dados exclusivamente foram recentemente propostos em [6].

\footnotetext{
Abstract: This work presents a methodology to obtain Hammerstein and Wiener models from priorly indentified NARX polynomial models. Analysis about polynomial expansion of block oriented models is carried out in order to specufy some of them porpertie. This approach is aplied on DC-DC Buck conversor as an example.
} 


\section{Referências}

[1] L.A. Aguirre, "Introdução à Identificação de Sistemas Técnicas Lineares e NãoLineares Aplicadas a Sistemas Reais", Editora da UFMG, Belo Horizonte, MG, 2000 .

[2] L.A. Aguirre and E.M.A.M. Mendes, Global nonlinear polynomial models: structure, term cluster and fixed points, Int. J. Bif. Chaos, 6, No. 2 (1996), 279-294.

[3] E.W. Bai, A blind approach to the Hammerstein-Wiener model identification, Automatica, 38 (2002), 967-979.

[4] S.A. Billings and I.J. Leontaritis, Parameter estimation techniques for nonlinear systems, em "6th IFAC Symposium on Identification and System Parameter Estimation", Washington D.C., USA, pp. 505-510, 1982.

[5] S. Chen and S.A. Billings, Representations of non-linear systems the NARMAX model, nt. J. Control, 49, No. 3 (1989), 1013-1032

[6] M.C.S. Coelho, "Modelos de Hammerstein e de Wiener: Conexões com Modelos NARX Polinomiais e Sua Aplicação em Identificação de Sistemas NãoLineares", Dissertação de mestrado, PPGEE, UFMG, Belo Horizonte, Brasil, (http://www.cpdee.ufmg.br/ ${ }^{\sim}$ MACSIN), 2002.

[7] M.V. Corrêa, "Identificação Caixa-Cinza de Sistemas Não-Lineares Utilizando Representações NARMAX Racionais e Polinomiais", Tese de Doutorado, PPGEE, UFMG, Belo Horizonte, MG, Brasil, 2001.

[8] E. Eskinat, S.H. Johnson, and W.L. Luyben, Use of Hammerstein models in identification nonlinear systems, AIChE Journal, 37, No. 2 (1991).

[9] K.P. Fruzzetti, A. Palazoglu and K.A. McDonald, Nonlinear model predictive control using Hammerstein models, Journal of process control, 7, No. 1, (1997), $31-41$

[10] I.W. Hunter and M.J. Korenberg, The identification of nonlinear biological systems: Wiener and Hammerstein cascade models, Biological Cybernetics, 50, No. 23 (1986), 3693-3701.

[11] I.J. Leontaritis and S.A. Billings, Input-output parametric models for nonlinear systems - part i: deterministic non-linear systems; Input-output parametric models for non-linear systems - part ii: sthocastic non-linear systems, Int. J. Control, 41, No. 2 (1985), 303-344.

[12] R.K. Pearson and M. Pottman, Gray-box identification of block oriented nonlinear models, Journal of Process Control, 10, No. 4 (2000), 301-315. 